\title{
FIGO Stage IV Gestational Trophoblastic
} Tumor

National Cancer Institute

\section{Source}

National Cancer Institute. FIGO Stage IV Gestational Trophoblastic Tumor. NCI

Thesaurus. Code C6404.

Gestational trophoblastic tumor in all other metastatic sites. 